Non-linear gyrokinetic simulations of microturbulence in TCV electron internal transport barriers

This article has been downloaded from IOPscience. Please scroll down to see the full text article.

2011 Plasma Phys. Control. Fusion 53054011

(http://iopscience.iop.org/0741-3335/53/5/054011)

View the table of contents for this issue, or go to the journal homepage for more

Download details:

IP Address: 128.178.125.186

The article was downloaded on 08/04/2011 at 08:35

Please note that terms and conditions apply. 


\title{
Non-linear gyrokinetic simulations of microturbulence in TCV electron internal transport barriers
}

\author{
X Lapillonne ${ }^{1}$, S Brunner ${ }^{1}$, O Sauter ${ }^{1}$, L Villard ${ }^{1}$, E Fable ${ }^{2}$, T Görler ${ }^{2}$, \\ F Jenko ${ }^{2}$ and F Merz ${ }^{2}$ \\ ${ }^{1}$ Centre de Recherches en Physique des Plasmas, Association EURATOM-Confédération Suisse, \\ Ecole Polytechnique Fédérale de Lausanne, CH-1015 Lausanne, Switzerland \\ ${ }^{2}$ Max-Planck-Institut für Plasmaphysik, EURATOM Association, Boltzmannstrasse 2, D-85748 \\ Garching, Germany \\ E-mail: stephan.brunner@epfl.ch
}

Received 15 September 2010, in final form 8 December 2010

Published 7 April 2011

Online at stacks.iop.org/PPCF/53/054011

\begin{abstract}
Using the local (flux-tube) version of the Eulerian code GENE (Jenko et al 2000 Phys. Plasmas 7 1904), gyrokinetic simulations of microturbulence were carried out considering parameters relevant to electron-internal transport barriers (e-ITBs) in the TCV tokamak (Sauter et al 2005 Phys. Rev. Lett. 94 105002), generated under conditions of low or negative shear. For typical density and temperature gradients measured in such barriers, the corresponding simulated fluctuation spectra appears to simultaneously contain longer wavelength trapped electron modes (TEMs, for typically $k_{\perp} \rho_{\mathrm{i}}<0.5$, $k_{\perp}$ being the characteristic perpendicular wavenumber and $\rho_{\mathrm{i}}$ the ion Larmor radius) and shorter wavelength ion temperature gradient modes (ITG, $k_{\perp} \rho_{\mathrm{i}}>$ $0.5)$. The contributions to the electron particle flux from these two types of modes are, respectively, outward/inward and may cancel each other for experimentally realistic gradients. This mechanism may partly explain the feasibility of e-ITBs. The non-linear simulation results confirm the predictions of a previously developed quasi-linear model (Fable et al 2010 Plasma Phys. Control. Fusion 52 015007), namely that the stationary condition of zero particle flux is obtained through the competitive contributions of ITG and TEM. A quantitative comparison of the electron heat flux with experimental estimates is presented as well.
\end{abstract}

(Some figures in this article are in colour only in the electronic version)

\section{Introduction}

Internal transport barriers (ITBs) [1,2] are regions of reduced outward radial energy and/or particle transport in the core of magnetic fusion relevant plasmas. This reduced transport is 
reflected by increased density and temperature gradients in the barrier region compared with standard discharges. ITBs may therefore provide a promising approach for achieving high performance regimes in fusion reactors. A true understanding of the physical processes that lead to the formation of ITBs is still lacking today and thus presents a major challenge to both theory and numerical modeling. Gyrokinetic codes, which enable us to simulate the turbulent transport in magnetic confinement plasmas, should provide a powerful tool to meet this goal. However, before tackling the actual problem of barrier formation, numerical simulations should first prove to be successful at reproducing the reduced outward radial transport of already formed barriers, given the corresponding experimentally measured background conditions, i.e. in particular, magnetic equilibrium geometry, as well as radial profiles of density $n$ and temperature $T$ for the different species.

Attempting to model the low transport levels for given experimental conditions is thus the motivation of this study, considering measurements obtained for electron-internal transport barriers (e-ITBs) in the TCV tokamak. Such electron barriers have been systematically obtained in TCV under conditions of low or negative magnetic shear $\hat{s}$, leading simultaneously to sharp density and electron temperature gradients [3-7]. Although heating (in particular, electron cyclotron resonance heating, ECRH) is applied to the core of the plasma, no external particle fueling is provided to this central region in these discharges. The e-ITBs are thus maintained during the discharge over hundreds of diffusion confinement times with a zero radial electron particle flux, which in itself is a remarkable feature of these barriers [6].

The e-ITBs in the TCV tokamak are characterized by (1) their high global energy confinement improvement, a factor of 4-6, over TCV L-mode scaling, (2) their high local electron temperature gradients, $R / L_{T_{\mathrm{e}}} \gtrsim 15$, compared with usual values in electron cyclotron (EC) heated discharges, for which typically $R / L_{T_{\mathrm{e}}} \simeq 10$, and (3) their high bootstrap current fraction (40-100\%) [3-7]. Here and in the following, $L_{A}$ stands for the characteristic gradient length of profile $A$ and $R$ for the major radius of the tokamak. It has been shown experimentally that the degree of magnetic shear reversal is the key to the formation and sustainment of the e-ITBs and furthermore that they can be maintained with zero loop voltage [3-5]. One can thus conclude that the improved density gradient in these barriers is not due to a Ware pinch effect [6]. Simulations of TCV e-ITBs using the ASTRA transport code have confirmed that the confinement improvement indeed increases with an increased negative magnetic shear profile [7].

In certain types of ITBs, a significant background $E \times B$ shearing rate appears as an essential characteristic. This seems not to be the case in the TCV e-ITBs. Indeed, although there is currently no systematic measurement of this background flow shearing rate, preliminary measurements [8] indicate that they are not more significant in e-ITB discharges than in standard Ohmic discharges and thus do not play an essential role in barrier formation and sustainment.

In terms of gyrokinetic modeling, the TCV e-ITBs have only been studied so far using the linear, global code LORB5 [9] and a quasi-linear model based on linear, local (flux-tube) gyrokinetic results from the GS2 code [10,11]. The former study, based on mixing length estimates, has shown that the stabilization of trapped electron modes (TEMs) by $(s-\alpha)$ effects provides an explanation for the reduced heat transport in the barriers. The latter study enabled us to show that the stabilization of TEMs can furthermore explain the significant electron thermo-diffusive pinch and resulting density peaking observed, albeit with strong central electron heating [6]. More specifically, the quasi-linear model presented in [11] has shown how the interplay of ion temperature gradient (ITG) and TEMs may lead to a zero electron particle flux for the correct combination $\left(R / L_{n_{\mathrm{e}}}, R / L_{T_{\mathrm{e}}}, R / L_{T_{\mathrm{i}}}\right)$ of normalized electron density, electron temperature and ion temperature gradients, respectively. In this model, the two types of modes provide different contributions to an effective inward particle 
pinch, resulting in part from a thermo-diffusive mechanism related to the radial temperature gradient $\partial T_{\mathrm{e}} / \partial \rho_{\mathrm{t}}$, which compensates for the outward diffusion related to the density gradient $\partial n_{\mathrm{e}} / \partial \rho_{\mathrm{t}}$. Here $\rho_{\mathrm{t}}=\sqrt{\phi_{\mathrm{t}} / \phi_{\mathrm{t}, \text { edge }}}$, with $\phi_{\mathrm{t}}$ the toroidal flux, is used as the definition of the local normalized minor radius.

Non-linear gyrokinetic simulations modeling TCV e-ITBs have, however, not been performed so far, which is the purpose of this work. The constraint that there is a significant density peaking in these barriers with no particle source is actually very strong. This study has thus focused on attempting to simulate the improved heat transport, characterized by a high $R / L_{T_{\mathrm{e}}}$, while satisfying the zero particle flux constraint with a significant density gradient, which is more demanding than studying the heat flux alone. Indeed, a large electron heat flux could sustain the observed $R / L_{T_{\mathrm{e}}}$ value. However, at such large $R / L_{T_{\mathrm{e}}}$, TEMs are destabilized already at small values of $R / L_{n_{\mathrm{e}}}$, which tends to result in a so-called 'pump-out' effect [11, 12]. This is why it is important to test the quasi-linear predictions [11] for the e-ITBs with nonlinear simulations as well, namely that e-ITBs can have both large $R / L_{T_{\mathrm{e}}}$ and $R / L_{n_{\mathrm{e}}}$ values with dominant electron heating.

In the following, it will be shown how the predictions of the quasi-linear model [11] for achieving a stationary electron particle flux, $\Gamma_{\mathrm{e}}=0$, are validated under the particular conditions relevant to the TCV e-ITB discharges by non-linear gyrokinetic simulations using the local (flux-tube) version of the GENE code [13]. Preliminary attempts at quantitative comparisons between simulation results and experimental measurements of the electron heat transport will be discussed as well. It will be shown that the local flux-tube simulation is unable to fully reproduce the low heat transport levels in e-ITBs. Indeed, the electron heat fluxes predicted by the simulations remain larger by at least a factor of two, even for electron gradient values $R / L_{n_{\mathrm{e}}}$ and $R / L_{T_{\mathrm{e}}}$ at the lower range of those measured in these barriers. It is thus concluded that the stabilization from finite size effects (measured by $\rho^{\star}=\rho_{\mathrm{i}} / a, \rho_{\mathrm{i}}$ being the ion Larmor radius, $a$ the minor radius of the plasma) are expected to be essential and thus that global simulations are required for a fully realistic modeling of e-ITBs. Identifying the limits of local flux-tube simulations for modeling the e-ITBs may thus be considered as an essential prerequisite in view of such global calculations.

The remainder of this paper is organized as follows: section 2 briefly describes the typical physical conditions observed in TCV e-ITB discharges. Section 3 summarizes the numerical model considered by the gyrokinetic GENE code. Results from linear simulations for e-ITB-type conditions are presented in section 4, and corresponding quasi-linear estimates for the particle flux in section 5. The non-linear simulation results for both particle and heat fluxes are then presented in section 6. Conclusions are drawn in section 7.

\section{2. e-ITBs in the TCV tokamak}

The TCV tokamak is characterized by a major radius $R=0.88 \mathrm{~m}$, minor radius $a=0.25 \mathrm{~m}$ (at mid-plane), a magnetic field on axis $B_{0}=1.44 \mathrm{~T}$, and a plasma current up to $I=1.0 \mathrm{MA}$. One of its particularities is its significant available auxiliary EC heating and current drive power provided by nine gyrotron sources totaling 4.5 MW. Making use of these sources, fully EC driven, bootstrap dominated, reversed magnetic shear e-ITB discharges have been systematically obtained [3-5]. Typical electron temperature $T_{\mathrm{e}}$ and density $n_{\mathrm{e}}$ profiles, as well as safety factor $q_{\mathrm{s}}$ and corresponding magnetic shear $\hat{s}$ profiles, obtained in such e-ITB experiments, are shown in figures $1(a)-(d)$, respectively, for TCV discharge \#29866 [7]. Delimited with vertical lines is the barrier region $0.28 \lesssim \rho_{\mathrm{t}} \lesssim 0.46$, clearly characterized by steep electron profile gradients. Note, in particular, that the magnetic shear inversion $(\hat{s}=0)$ falls within the barrier at $\rho_{\mathrm{t}} \simeq 0.4$. The measured electron gradients in the e-ITBs are typically 

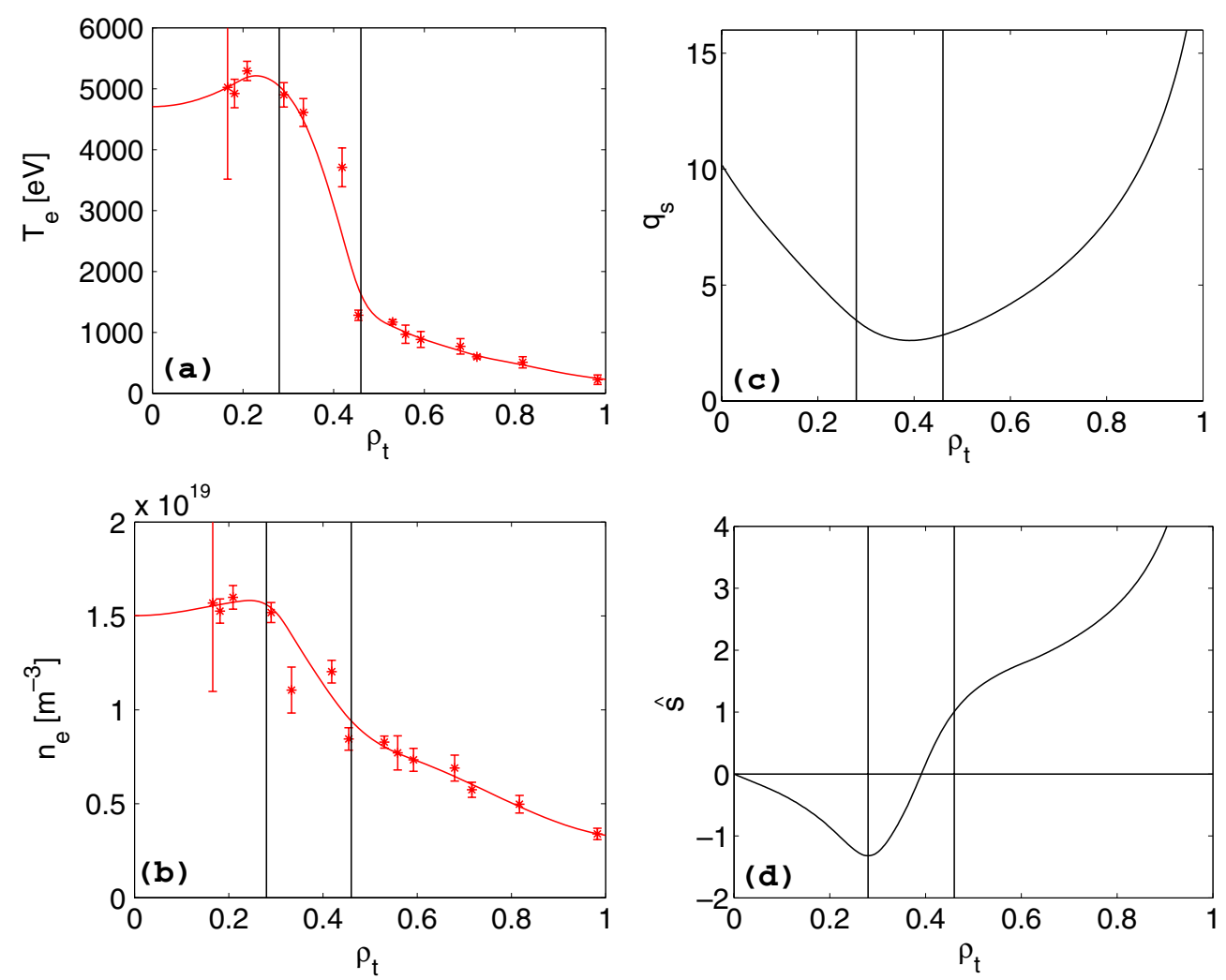

Figure 1. TCV discharge \#29866 with e-ITB in radial region $0.28 \lesssim \rho_{\mathrm{t}} \lesssim 0.46$. Shown profiles are the experimentally measured $(a)$ electron temperature $T_{\mathrm{e}}$ and $(b)$ electron density $n_{\mathrm{e}}$, as well as $(c)$ safety factor $q_{\mathrm{s}}$ and $(d)$ magnetic shear $\hat{s}$ from MHD reconstruction provided by CHEASE.

in the range $R / L_{T_{\mathrm{e}}}=10-30$ for temperature profiles and $R / L_{n_{\mathrm{e}}}=3-15$ for density profiles. Based on a limited number of other TCV discharges using charge exchange recombination spectroscopy (CXRS), the electron/ion temperature ratio is estimated at $\tau=T_{\mathrm{e}} / T_{\mathrm{i}}=3.5$. No accurate ion temperature profile measurements are, however, available so far for the here considered e-ITB discharges, so that $R / L_{T_{\mathrm{i}}}$ remains unknown. The effective ionization degree is approximately $Z_{\text {eff }} \simeq 2$, with carbon $\left(Z_{\mathrm{C}}=6\right)$ as dominant impurity.

\section{Numerical model}

The GENE code [13] was used for carrying out the gyrokinetic simulations. GENE is an Eulerian-based code, i.e. discretizing the distributions of the different plasma species on a fixed Cartesian grid in phase space, and enables non-linear simulations of microturbulence in both tokamak and stellarator geometries. Full multi-species kinetic dynamics, as well as electrostatic and electromagnetic fluctuations, may be considered. Linearized self- and inter-species collisions are also implemented. Interfacing to MHD equilibrium codes such as CHEASE [14] is provided. The five-dimensional phase space variables considered in GENE are the three field-aligned configuration space coordinates $(x, y, z)$ and the two velocity variables $\left(v_{\|}, \mu\right)$. Here $x$ stands for the radial, $y$ the bi-normal and $z$ the so-called 
parallel coordinate (corresponding, in fact, to the straight field line poloidal angle, used for labeling the position along the magnetic line), while $v_{\|}$is the parallel velocity, and $\mu=m v_{\perp}^{2} / 2 B$ the magnetic moment. GENE is massively parallelized using domain decomposition over all phase space directions as well as over the kinetic species. For linear mode analysis, it makes use of the PETSc/SLEPC package $[15,16]$ for eigenvalue computations.

More recently, GENE has been generalized for tokamak plasmas from a flux-tube to a global geometry [17-19], with the goal of addressing finite $\rho^{\star}$ effects. The global GENE version, in particular, includes radial variation of profiles (density, temperature, geometrical coefficients), non-periodic radial boundaries, as well as particle and heat sources/sinks which thus enable us to carry out global, quasi-stationary simulations of microturbulence.

In order to model conditions relevant to e-ITBs observed in TCV, discharge \#29866 described in section 2 is considered. Although global simulations might turn out to be essential for fully modeling e-ITBs, this study was mainly carried out with the flux-tube version of GENE. These local simulations have been run for the physical parameters at the particular radial position $\rho_{\mathrm{t}}=0.3$, corresponding to a local inverse aspect ratio $\epsilon=r / R=0.09$. The computations make use of the magnetic equilibrium reconstruction provided by CHEASE, in particular, giving the safety factor and shear values $q_{\mathrm{s}}=3.2$ and $\hat{s}=-1.17$, respectively, at $\rho_{\mathrm{t}}=0.3$. Three kinetic species with real mass ratios are evolved: electrons $\left(\mathrm{e}^{-}\right)$, deuterium $\left(\mathrm{D}^{+}\right)$and carbon $\left(\mathrm{C}^{6+}\right)$ as impurities. The following physical parameters are set for the different species: for electrons, the density and temperature gradient values $R / L_{n_{\mathrm{e}}}=3$ and $R / L_{T_{\mathrm{e}}}=12$ are chosen. Note that these electron gradient values are at the lower end but nonetheless within the corresponding ranges measured in the TCV e-ITBs. For the electron density and temperature, used for estimating collisionality, the values $n_{\mathrm{e}}=1.2 \times 10^{19} \mathrm{~m}^{-3}$ and $T_{\mathrm{e}}=4.5 \mathrm{keV}$ are considered. Both ion species $\left(\mathrm{D}^{+}\right.$and $\left.\mathrm{C}^{6+}\right)$ are assumed to have identical temperature profiles, $T_{\mathrm{D}} \equiv T_{\mathrm{C}} \equiv T_{\mathrm{i}}$, with an electron to ion temperature ratio $\tau=T_{\mathrm{e}} / T_{\mathrm{i}}=3.5$. The unknown ion temperature gradients are scanned over the range $R / L_{T, \mathrm{D}}=R / L_{T, \mathrm{C}}=$ $R / L_{T_{\mathrm{i}}}=2-10$. The ion/electron density ratios are set to $n_{\mathrm{D}} / n_{\mathrm{e}}=0.8$ and $n_{\mathrm{C}} / n_{\mathrm{e}}=0.03$, ensuring both quasi-neutrality, i.e. $n_{\mathrm{e}}=n_{\mathrm{D}}+Z_{\mathrm{C}} n_{\mathrm{C}}$, and $Z_{\mathrm{eff}}=\sum_{i} n_{\mathrm{i}} Z_{\mathrm{i}}^{2} / \sum_{i} n_{\mathrm{i}} Z_{\mathrm{i}}=2$ (where the sums are over all ion species), in agreement with the experimental measurements. The ion density gradients are themselves constrained by quasi-neutrality, leading to the relation $\left(n_{\mathrm{D}} / n_{\mathrm{e}}\right)\left(R / L_{n \mathrm{D}}\right)+Z_{\mathrm{C}}\left(n_{\mathrm{C}} / n_{\mathrm{e}}\right)\left(R / L_{n \mathrm{C}}\right)=R / L_{n_{\mathrm{e}}}$. Furthermore, a study carried out in [20], based on the same quasi-linear model as in [10], has shown that for $R / L_{n \mathrm{C}} \simeq(1 / 2) R / L_{n_{\mathrm{e}}}$ both the electron and the carbon impurity particle fluxes could be simultaneously canceled, i.e. $\Gamma_{\mathrm{e}}=\Gamma_{\mathrm{C}}=0$. These two constraints were thus used for estimating the density gradient values $R / L_{n \mathrm{D}}=3.4$ and $R / L_{n \mathrm{C}}=1.5$, for deuterium and carbon, respectively. The finite collisionality is accounted for in the simulations and is based on the experimental values, leading to a normalized collisionality $\nu_{\mathrm{e}}^{\star}=1 / \tau_{\mathrm{e}} \omega_{\mathrm{t}, \mathrm{e}} \epsilon^{3 / 2}=5.6 \times 10^{-2}$, where $\tau_{\mathrm{e}}=3(2 \pi)^{3 / 2} \epsilon_{0}^{2} T_{\mathrm{e}}^{3 / 2} m_{\mathrm{e}}^{1 / 2} / n_{\mathrm{i}} Z^{2} e^{4} \log \Lambda$ is the electron collision time, $\omega_{\mathrm{t}, \mathrm{e}}=v_{\mathrm{th}, \mathrm{e}} / R q_{\mathrm{s}}$ the transit frequency, $v_{\text {th }}=(T / m)^{1 / 2}$ the thermal velocity, $m_{\mathrm{e}}$ the electron mass, and having chosen the value $\log \Lambda=20$ for the Coulomb logarithm. A finite $\beta=10^{-4}$ is considered, which is small compared with the experimental value $\beta \simeq 10^{-2}$, but enables us to suppress the high-frequency electrostatic shear-Alfvèn modes [21] (thus avoiding the correspondingly required small time steps) while avoiding electromagnetic effects on the transport, so that the simulations may be considered as essentially electrostatic. Note that the temperature ratio $T_{\mathrm{e}} / T_{\mathrm{i}}>1$ as well as $Z_{\text {eff }}>1$ both have a stabilizing effect on the very short wavelength electron temperature gradient (ETG) modes [22], which thus did not need to be resolved. 

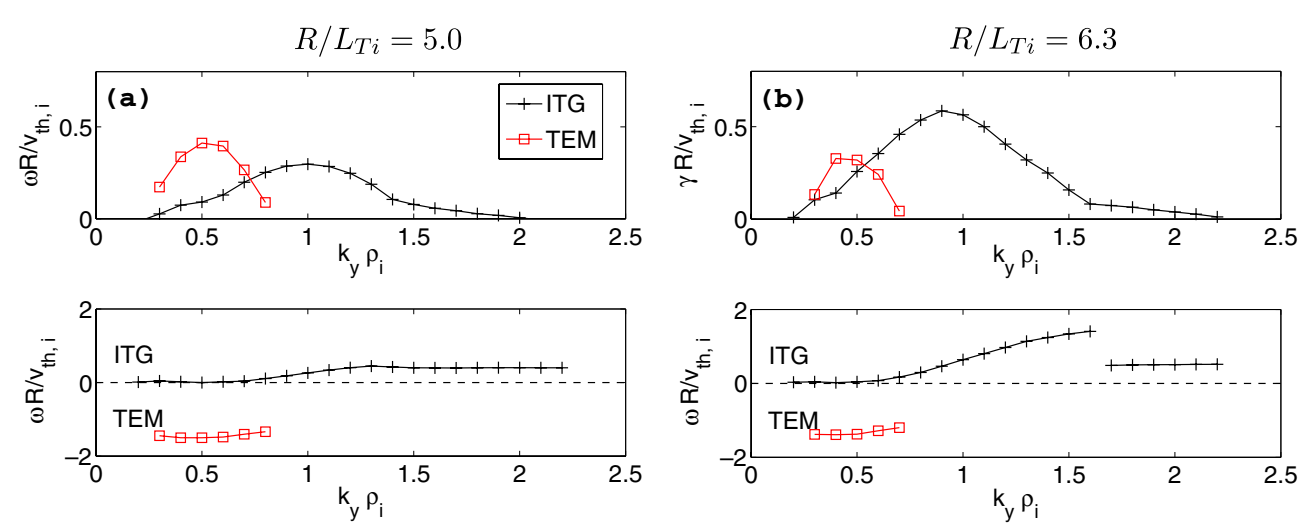

Figure 2. Linear frequencies and growth rates of most unstable modes as a function of $k_{y} \rho_{\text {i }}$ for (a) $R / L_{T_{\mathrm{i}}}=5.0$ and $(b) R / L_{T_{\mathrm{i}}}=6.3$.

\section{Linear simulations}

Considering the two different normalized ion temperature gradients $R / L_{T_{\mathrm{i}}}=5.0$ and $R / L_{T_{\mathrm{i}}}=6.3$, the GENE code was first run in its spectral analysis mode to obtain the most unstable eigenmodes for each wavenumber $k_{y} \rho_{\mathrm{i}}$ over the range $0.0<k_{y} \rho_{\mathrm{i}}<2.2$. The deuterium Larmor radius is defined as $\rho_{\mathrm{i}}=v_{\mathrm{th}, \mathrm{i}} / \Omega_{\mathrm{i}}$, with $\Omega_{\mathrm{i}}=e B_{0} / m_{\mathrm{i}}$ the cyclotron frequency. Given the definition of the $y$ coordinate considered in GENE, the corresponding wavenumber can be written as $k_{y}=n q_{\mathrm{s}} / r$, where $n$ is the toroidal wavenumber and $r=\rho_{\mathrm{t}} a$ is the minor radius at the flux-tube position. The linear growth rates $\gamma$ and real frequencies $\omega_{\mathrm{R}}$ as a function of $k_{y} \rho_{\mathrm{i}}$, normalized with respect to $v_{\mathrm{th}, \mathrm{i}} / R$, are shown in figure 2. For both values of $R / L_{T_{\mathrm{i}}}$, the unstable $k_{y}$ spectra simultaneously contains longer wavelength TEM modes, i.e. with negative frequency, and shorter wavelength ITG modes, with positive frequency. More specifically, the most unstable eigenmode for each $k_{y}$ is a TEM for $k_{y}<k_{0}$ and an ITG for $k_{y}>k_{0}$. Note, however, that going from $R / L_{T_{\mathrm{i}}}=5.0$ to $R / L_{T_{\mathrm{i}}}=6.3$ the most unstable mode over the whole spectrum changes from TEM to ITG and the transition wavenumber shifts down from $k_{0} \rho_{\mathrm{i}}=0.7$ to $k_{0} \rho_{\mathrm{i}}=0.5$. Finally, for the ion temperature gradient $R / L_{T_{\mathrm{i}}}=6.3$ considered in figure $2(b)$, there are in fact two unstable ITG branches that coexist simultaneously for $k_{y} \rho_{\mathrm{i}}>1$; however, only the frequency and growth rate of the most unstable ITG mode at each $k_{y}$ has been shown in this plot. The discontinuity in the real frequency at $k_{y} \rho_{\mathrm{i}}=1.6$ corresponds to the wavenumber at which the growth rates of these two branches cross.

\section{Quasi-linear study}

Quasi-linear estimates of fluxes are obtained using the same model as considered in [11]. The quasi-linear flux $F^{\text {q.l. }}$, where $F$ stands either for the electron particle flux $\Gamma_{\mathrm{e}}$ or heat flux $Q_{\mathrm{e}}$ is thus computed as follows:

$$
F^{\mathrm{q} .1 .}=\sum_{k_{y}} \hat{F}_{k_{y}}^{\mathrm{q} .1 .} \Delta k_{y},
$$

where $\Delta k_{y}$ is the wavenumber spacing and the contribution from the mode $k_{y}$ is estimated by

$$
\hat{F}_{k_{y}}^{\mathrm{q} .1 .}=\frac{A\left(k_{y}\right)}{\left|\hat{\Phi}_{0, k_{y}}(0)\right|^{2}} \hat{F}_{k_{y}} \text {. }
$$


The term $\hat{F}_{k_{y}}$ stands for the linear flux, which is computed from the most unstable eigenmode provided by linear GENE simulations at each $k_{y}$. For particle and heat fluxes, respectively, $\hat{F}_{k_{y}}$ is thus given by

$$
\begin{aligned}
& \hat{\Gamma}_{k_{y}}=\left\langle 2 \Re \sum_{k_{x}}\left[\frac{\mathrm{i} k_{y}}{B_{0}} \int \mathrm{d}^{3} v \delta \hat{f}_{k_{x}, k_{y}} \hat{\bar{\Phi}}_{k_{x}, k_{y}}^{\star}\right]\right\rangle_{z}, \\
& \hat{Q}_{k_{y}}=\left\langle 2 \Re \sum_{k_{x}}\left[\frac{\mathrm{i} k_{y}}{B_{0}} \int \mathrm{d}^{3} v \frac{m}{2} v^{2} \delta \hat{f}_{k_{x}, k_{y}} \hat{\bar{\Phi}}_{k_{x}, k_{y}}^{\star}\right]\right\rangle_{z} .
\end{aligned}
$$

In (3) and (4), $\langle A\rangle_{z}=\int J^{x y z}(z) A(z) \mathrm{d} z / \int J^{x y z}(z) \mathrm{d} z$ completes the flux surface-averaging, with $J^{x y z}$ the Jacobian relative to the coordinate system $(x, y, z), \Re$ takes the real part of the following complex number and $\left[\delta \hat{f}_{k_{x}}, k_{y}(z), \hat{\bar{\Phi}}_{k_{x}}, k_{y}(z)\right]$ are the $\left(k_{x}, k_{y}\right)$ Fourier components of the distribution fluctuation and gyro-averaged electrostatic potential, respectively. In Fourier space, the gyro-averaged field is conveniently obtained from the relation $\hat{\bar{\Phi}}_{k_{x}, k_{y}}=$ $J_{0}\left(k_{\perp} v_{\perp} / \Omega\right) \hat{\Phi}_{k_{x}, k_{y}}$, where $J_{0}$ is the zeroth order Bessel function, $\Omega$ the cyclotron frequency, $k_{\perp}^{2}=g^{x x} k_{x}^{2}+2 g^{x y} k_{x} k_{y}+g^{y y} k_{y}^{2}$, and $g^{\mu, v}=\nabla \mu \cdot \nabla v$ the geometric coefficients related to the coordinate system $(x, y, z)$. The amplitudes of linear modes being physically irrelevant, the linear fluxes must be renormalized, which is the role of the factor $A\left(k_{y}\right) /\left|\hat{\Phi}_{0, k_{y}}(0)\right|^{2}$ appearing in (2), where the spectral amplitude weighting $A\left(k_{y}\right)$ is given for the considered quasi-linear model [11] by

$$
A\left(k_{y}\right)=A_{0}\left(\frac{\gamma_{k_{y}}}{\left\langle k_{\perp}^{2}\right\rangle}\right)^{2} .
$$

In (5), $\gamma_{k_{y}}$ is the linear growth rate of the most unstable mode at $k_{y}$ and the average squared perpendicular wavenumber $\left\langle k_{\perp}^{2}\right\rangle$ is obtained by weighting over the corresponding eigenmode envelope $\hat{\Phi}_{k_{x}, k_{y}}(z)$ :

$$
\left\langle k_{\perp}^{2}\right\rangle=\frac{\sum_{k_{x}} \int\left(g^{x x} k_{x}^{2}+2 g^{x y} k_{x} k_{y}+g^{y y} k_{y}^{2}\right)\left|\hat{\Phi}_{k_{x}, k_{y}}(z)\right|^{2} \mathrm{~d} z}{\sum_{k_{x}} \int\left|\hat{\Phi}_{k_{x}, k_{y}}(z)\right|^{2} \mathrm{~d} z} .
$$

Figure $3(a)$ plots the spectral density for the quasi-linear electron particle flux $\hat{\Gamma}_{\mathrm{e}}^{\mathrm{q} .1 .}\left(k_{y}\right)$ as a function of $k_{y}$ for the different ion temperature gradients $R / L_{T_{\mathrm{i}}}=5.0,6.3$ and 7.5. This spectral analysis shows (positive) outward particle flux contributions at the lower $k_{y}$ values $\left(k_{y}<k_{0}\right)$ where TEM modes are most unstable, while (negative) inward flux contributions are provided at the higher $k_{y}$ values $\left(k_{y}>k_{0}\right)$ where the ITG modes are most unstable (see figure 2). This result stresses the importance of keeping all $k_{y}$ mode contributions in the quasilinear estimates (1) of the fluxes. According to the theoretical study of possible turbulent convective mechanisms carried out in [11] in the frame of the quasi-linear model, the outward (respectively inward) fluxes in the case of TEM (respectively ITG) modes can be identified as a thermo-diffusive pinch effect resulting from the trapped particles whose precessional drift frequency resonates with the wave frequency. One should point out that the condition of zero particle flux, coinciding with the ITG/TEM transition, has also been identified using other types of quasi-linear models, such as described e.g. in [23, 24].

The quasi-linear estimates for the total electron particle flux $\Gamma_{\mathrm{e}}^{\mathrm{q} .1 .}$ are plotted in figure 3(b) as a function of $R / L_{T_{\mathrm{i}}}$, clearly showing a transition from an effectively outward flux at lower ion temperature gradients where the TEM is the overall most unstable mode to an inward flux for higher gradients where the ITG is the overall most unstable. A stationary state with zero particle flux, $\Gamma_{\mathrm{e}}=0$, is thus predicted at the so-called stationary gradient $R / L_{T_{\mathrm{i}} \text {,stat }}$. The estimate by the quasi-linear model for this stationary gradient is thus $R / L_{T_{\mathrm{i}} \text {, stat }}=6.5$. 

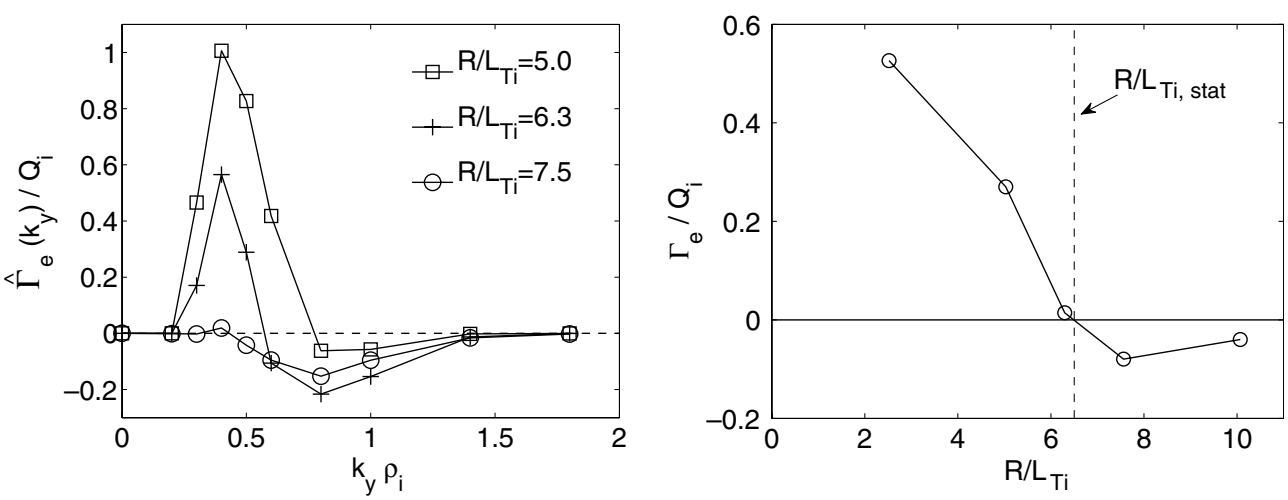

Figure 3. Results from the quasi-linear model: $(a)$ electron particle flux spectra $\hat{\Gamma}_{\mathrm{e}}^{\mathrm{q} .1 .}\left(k_{y}\right)$ for different $R / L_{T_{\mathrm{i}}}$ and $(b)$ total particle flux $\Gamma_{\mathrm{e}}^{\mathrm{q} .1 .}$ versus $R / L_{T_{\mathrm{i}}}$. Note the stationary gradient $R / L_{T_{\mathrm{i}} \text {,stat }}$ for which $\Gamma_{\mathrm{e}}=0$. Particle fluxes have been normalized with respect to the total deuterium heat flux $Q_{\mathrm{i}}$.
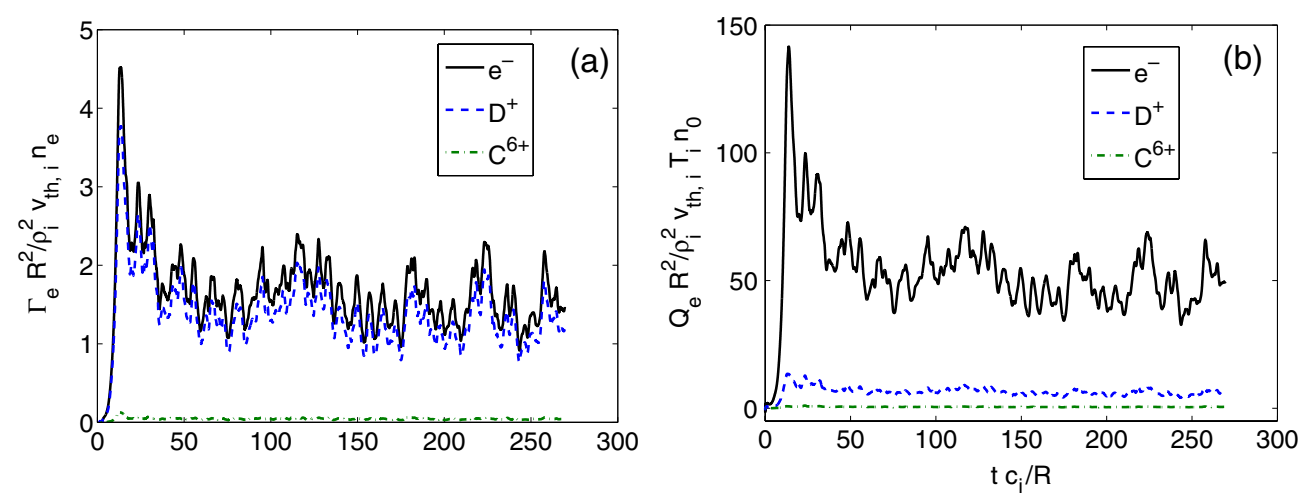

Figure 4. Non-linear simulation results: Time evolution of fluxes of the different species (electrons $\left(\mathrm{e}^{-}\right)$, deuterium $\left(\mathrm{D}^{+}\right)$, carbon $\left(\mathrm{C}^{6+}\right)$ from non-linear simulation at $R / L_{T_{\mathrm{i}}}=5.0$. (a) Particle fluxes and $(b)$ heat fluxes.

Note that all particle fluxes shown in figure 3 have been normalized with respect to the total quasi-linear deuterium heat flux $Q_{\mathrm{i}}^{\mathrm{q} .1 .}$ so as to remove the calibration coefficient $A_{0}$ appearing in the spectral weighting (5).

\section{Non-linear simulations}

Non-linear GENE simulations were then carried out for the same e-ITB relevant conditions as those considered for the linear and quasi-linear studies. The time traces for the non-linear particle and heat fluxes for all three species, electrons, deuterium and carbon, are shown in figure 4 for the ion temperature gradient $R / L_{T_{\mathrm{i}}}=5.0$. The TEM is the most unstable linear mode for this value of $R / L_{T_{\mathrm{i}}}$ and, in agreement with the quasi-linear estimates, the total electron particle flux is outward. Note also that the electron heat flux dominates the ion heat fluxes in this case, which is also consistent with the fact that the TEM is the most unstable mode. One can furthermore verify that the particle fluxes for the different species are such that the ambipolarity condition $\Gamma_{\mathrm{D}}+Z_{\mathrm{C}} \Gamma_{\mathrm{C}}=\Gamma_{\mathrm{e}}$ is exactly satisfied. 

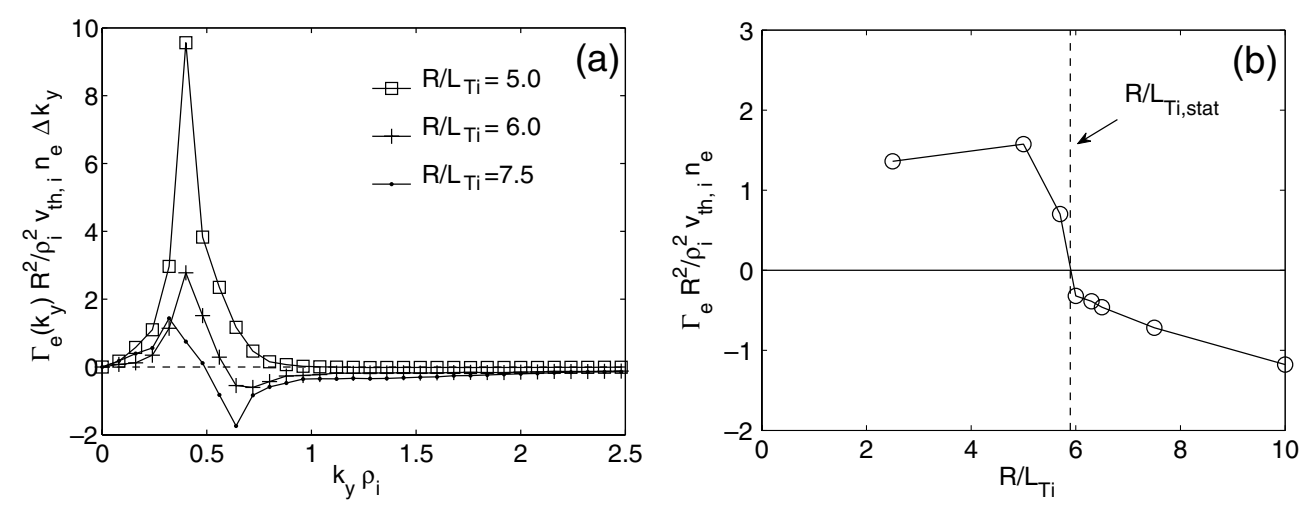

Figure 5. Non-linear simulation results: $(a)$ electron particle flux spectra $\Gamma_{\mathrm{e}}\left(k_{y}\right)$ for different $R / L_{T_{\mathrm{i}}}$ and $(b)$ total particle flux $\Gamma_{\mathrm{e}}$ versus $R / L_{T_{\mathrm{i}}}$.

The spectral density of the non-linear electron particle flux $\hat{\Gamma}_{\mathrm{e}}\left(k_{y}\right)$ is shown in figure $5(a)$ as a function of $k_{y}$ for essentially the same values of the ion temperature gradients $R / L_{T_{\mathrm{i}}}$ as considered for the quasi-linear results of figure 3(a). The total electron particle fluxes as a function of $R / L_{T_{\mathrm{i}}}$ are plotted in figure $5(b)$. These non-linear results clearly validate the quasi-linear model results, in particular, concerning the detailed spectral features of outward (respectively inward) fluxes in the linearly TEM (respectively ITG) dominated $k_{y}$ ranges. Furthermore, the stationary ion temperature gradient $R / L_{T_{\mathrm{i}} \text {, stat }}=5.9$ obtained from these non-linear simulation, i.e. where $\Gamma_{\mathrm{e}}=0$, is in good agreement with the quasi-linear estimate value $R / L_{T_{\mathrm{i}} \text {,stat }}=6.5$. This close agreement is also a strong indication that the mechanisms at play in the non-linear simulations which lead to the particle pinch are essentially the same as those which had been identified in the frame of the quasi-linear model [11].

As already mentioned, the ion gradient $R / L_{T_{\mathrm{i}} \text {, stat }}$ is of particular interest as it corresponds, from the particle transport point of view, to an equilibrium state for a system with no internal particle sources. In our case of interest, $R / L_{T_{\mathrm{i}} \text {, stat }}$ should thus agree with the ion temperature gradient in the fully developed e-ITB. The corresponding experimental value is, however, unavailable at this point. In an attempt to nonetheless further validate the relevance of the non-linear simulations for modeling the considered e-ITB, one may compare the electron heat transport computed for $R / L_{T_{\mathrm{i}} \text {, stat }}$ with experimental measurements obtained within the barrier. To this end, the spectral density of the non-linear electron heat diffusivity $\hat{\chi}_{\mathrm{e}}\left(k_{y}\right)$ as well as the total heat diffusivity $\chi_{\mathrm{e}}$ are shown in figure 6 , for the same values of $R / L_{T_{\mathrm{i}}}$ as in figure 5. The electron heat diffusivity is estimated here as $\chi_{\mathrm{e}}=Q_{\mathrm{e}} / n_{\mathrm{e}}\left\langle\left|\nabla T_{\mathrm{e}}\right|\right\rangle$, where $\left\langle\left\langle\left|\nabla T_{\mathrm{e}}\right|\right\rangle\right\rangle=\partial T_{\mathrm{e}} / \partial \rho_{\mathrm{t}}\left\langle\left|\nabla \rho_{t}\right|\right\rangle$ is the flux surface-averaged electron temperature gradient.

A remarkable feature which appears in the $R / L_{T_{\mathrm{i}}}$ scan of $\chi_{\mathrm{e}}$ is the well defined local minimum of the electron heat transport at $R / L_{T_{\mathrm{i}}}$,stat . A very similar effect had already been observed and discussed in [24]. From the spectral decomposition of the heat diffusivity in figure $6(a)$, one observes that the peak related to TEM modes at $k_{y} \rho_{\mathrm{i}} \simeq 0.4$ is removed when going from $R / L_{T_{\mathrm{i}}}=5.0$ to $R / L_{T_{\mathrm{i}}}=6.0$. A new peak then appears in the ITG part of the spectrum around $k_{y} \rho_{\mathrm{i}} \simeq 0.7$, which then increases together with the ion temperature gradient beyond $R / L_{T_{\mathrm{i}}}=6.0$. A nonlinear interaction between ITG and TEM modes in the spectral region $k_{y} \rho_{\mathrm{i}} \simeq 0.5$, where these two modes have similar linear growth rates, thus appears as a reasonable explanation for the local minimum of the electron heat flux around $R / L_{T_{\mathrm{i}} \text {,stat }}$. According to figure $6(b)$, the estimate from the non-linear simulations for the electron heat diffusivity at $R / L_{T_{\mathrm{i}} \text {, stat }}$ is thus of order $\chi_{\mathrm{e}}\left(R / L_{T_{\mathrm{i}} \text {, stat }}\right) \simeq 2 \mathrm{~m}^{2} \mathrm{~s}^{-1}$, which is to be compared with the experimentally measured value $\chi_{\mathrm{e}}^{\text {exp. }} \simeq 1 \mathrm{~m}^{2} \mathrm{~s}^{-1}$. The minimum of $\chi_{\mathrm{e}}$ at $R / L_{T_{\mathrm{i}} \text {, stat }}$, 

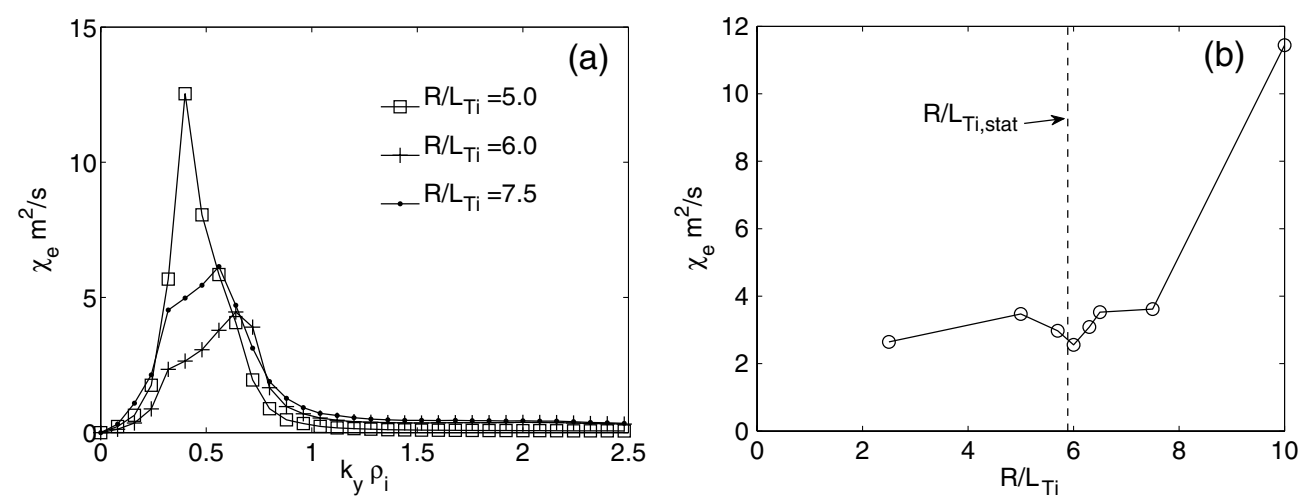

Figure 6. Same as in figure 5 but showing $(a)$ electron heat diffusivity spectra $\chi_{\mathrm{e}}\left(k_{y}\right)$ and $(b)$ total diffusivity $\chi_{\mathrm{e}}$ in SI units $\left(\mathrm{m}^{2} \mathrm{~s}^{-1}\right)$.

corresponding to a local relative reduction of $30 \%$ (the neighboring $R / L_{T_{\mathrm{i}}}$ dependence of $\chi_{\mathrm{e}}$ is quite flat and of order $\chi_{\mathrm{e}}=3.5 \mathrm{~m}^{2} \mathrm{~s}^{-1}$ ) is thus insufficient in itself in explaining the low heat transport observed in the barriers. One should nonetheless note that although still significantly larger by a factor of two, this numerical result for the electron heat transport is at least within the same order of magnitude as the experimental measurement.

\section{Conclusions}

Linear and non-linear flux-tube simulations using the GENE code have been carried out for conditions relevant to e-ITBs in the TCV tokamak. For the electron temperature and density gradients observed in these barriers, the simulation results show that a particular ion temperature gradient $R / L_{T_{\mathrm{i}}}$, stat may be found for which the electron particle flux $\Gamma_{\mathrm{e}}$ goes to zero. Such a state with $\Gamma_{\mathrm{e}}=0$ is expected to exist under conditions of no internal particle sources, as is the case in the e-ITB discharges of interest. The non-linear simulations clearly show how the zero electron particle flux results from the coexistence of TEM and ITG modes in the unstable $k_{y}$ spectrum, respectively, leading to outward and inward contributions which cancel out at $R / L_{T_{\mathrm{i}} \text {,stat }}$. These non-linear results strongly validate predictions obtained with the quasi-linear model considered in [11], in particular, concerning the detailed spectral features, as well as in quantitatively estimating the stationary gradient $R / L_{T_{\mathrm{i}} \text {, stat }}$.

Unfortunately, TCV lacks diagnostics for estimating ion temperature gradients and the value for the stationary gradient obtained from the simulations can therefore not be directly confronted to experimental measurements. Instead, the heat diffusivity $\chi_{\mathrm{e}}$ obtained from the non-linear flux-tube simulations at $R / L_{T_{\mathrm{i}} \text {,stat }}$ has been compared with the experimental values measured in the e-ITB and an agreement within a factor of two was found. It is to be noted, however, that the electron temperature and density gradients $R / L_{n_{\mathrm{e}}}=3$ and $R / L_{T_{\mathrm{e}}}=12$, which have been considered in the simulations presented here, are at the lower range of the experimental values observed in TCV e-ITBs (see section 2). A preliminary sensitivity study has been carried out in which stronger electron density and temperature gradients, i.e. in the middle range of measured values, were considered. In all cases, an ion temperature gradient $R / L_{T_{\mathrm{i}}}=R / L_{T_{\mathrm{i}} \text {, stat }}$ could still be identified for which the electron particle flux $\Gamma_{\mathrm{e}}$ canceled. However, the corresponding electron heat diffusivity $\chi_{\mathrm{e}}$ increased with these steepened gradients, especially with respect to $R / L_{n_{\mathrm{e}}}$, taking on values clearly above the experimental ones. 
Further work is thus clearly still required to fully explain the transport mechanisms in eITBs. One possible explanation is the finite $\rho^{\star}$ effects, which may be accentuated by the narrow gradient profiles in ITBs and may lead to significantly reduced transport levels compared with the ones predicted by local flux-tube simulations [25]. Investigating such finite size effects in TCV e-ITBs shall be studied in the near future making use of the recently developed global version of GENE. Finally, more realistic simulations should also allow for electromagnetic fluctuations by accounting for the finite $\beta$ values in the experiment, whose effect could be particularly significant in the large pressure gradient region of the e-ITBs.

\section{Acknowledgments}

This work was partly supported by the Swiss National Science Foundation. The simulations have been carried out on the Pleiades 2 cluster at the EPFL as well as on the HPC-FF computer at the Jülich Forschungszentrum, Germany.

\section{References}

[1] Wolf R C 2003 Plasma Phys. Control. Fusion 45 R1

[2] Connor J, Fukuda T, Garbet X, Gormezano C, Mukhovatov V and Wakatani M (the ITB Database Group and the ITPA Topical Group on Transport and Internal Barrier Physics) 2004 Nucl. Fusion 44 R1

[3] Henderson M A, Camenen Y, Coda S, Goodman T P, Nikkola P, Pochelon A and Sauter O (the TCV Team) 2004 Phys. Rev. Lett. 93215001

[4] Sauter O, Coda S, Goodman T P, Henderson M A, Behn R, Bottino A, Fable E, Martynov A, Nikkola P and Zucca C (the TCV Team) 2005 Phys. Rev. Lett. 94105002

[5] Goodman T P et al 2005 Plasma Phys. Control. Fusion 47 B107

[6] Fable E, Sauter O, Coda S, Goodman T P, Henderson M A, Weisen H, Zabolotsky A and Zucca C (the TCV Team) 2006 Plasma Phys. Control. Fusion 48 1271-83

[7] Zucca C, Sauter O, Asp E, Coda S, Fable E, Goodman T P and Henderson M A 2009 Plasma Phys. Control. Fusion $\mathbf{5 1} 015002$

[8] Sauter O 2010 Proc. IAEA Meeting (11-16 October 2010, Daejeon, Republic of Korea) EXS/P2-17

[9] Bottino A, Sauter O, Camenen Y and Fable E 2006 Plasma Phys. Control. Fusion 48 215-33

[10] Fable E, Angioni C and Sauter O 2008 Plasma Phys. Control. Fusion 50115005

[11] Fable E, Angioni C and Sauter O 2010 Plasma Phys. Control. Fusion 52015007

[12] Angioni C, Peeters A G, Garbet X, Manini A and Ryter F (the ASDEX Upgrade Team) 2004 Nucl. Fusion $44827-45$

[13] Jenko F, Dorland W, Kotschenreuther M and Rogers B N 2000 Phys. Plasmas 7 1904-10

[14] Lütjens H, Bondeson A and Sauter O 1996 Comput. Phys. Commun. 97 219-60

[15] Balay S, Buschelman K, Gropp W D, Kaushik D, Knepley M G, McInnes L C, Smith B F and Zhang H 2009 PETSc Web page http://www.mcs.anl.gov/petsc

[16] Hernandez V, Roman J E and Vidal V 2005 ACM Trans. Math. Softw. 31 351-62

[17] Görler T 2009 PhD Thesis University of Ulm, Germany

[18] Lapillonne X 2010 PhD Thesis Ecole Polytechnique Fédérale de Lausanne, Switzerland http://library.epfl. $\mathrm{ch} /$ theses/?nr=4684

[19] Görler T, Lapillonne X, Brunner S, Dannert T, Jenko F, Merz F and Told D J. Comput. Phys. Submitted for publication

[20] Fable E 2009 PhD Thesis Ecole Polytechnique Fédérale de Lausanne, Switzerland http://library.epfl.ch/ theses/?nr=4334

[21] Lee W W, Lewandowski J L V, Hahm T S and Lin Z 2001 Phys. Plasmas 8 4435-40

[22] Jenko F, Dorland W and Hammett G W 2001 Phys. Plasmas 8 4096-104

[23] Jenko F, Dannert T and Angioni C 2005 Plasma Phys. Control. Fusion 47 B195-B206

[24] Merz F and Jenko F 2010 Nucl. Fusion 50054005

[25] McMillan B F, Lapillonne X, Brunner S, Villard L, Jolliet S, Bottino A, Görler T and Jenko F 2010 Phys. Rev. Lett. 105155001 